\title{
Digestion of fat by young pigs: a study of the amounts of fatty acid in the digestive tract using a fat-soluble indicator of absorption
}

\author{
BY W. E. CARLSON* AND H. S. BAYLEY \\ Department of Nutrition, University of Guelph, Guelph, Ontario, Canada
}

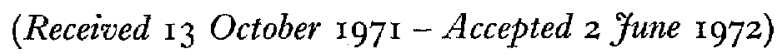

\begin{abstract}
I. The addition of $o_{1} \%$ tridodecyl glyceryl ether (DGE) to semi-purified diets, containing $10 \%$ beef tallow did not affect food intake, growth or fatty-acid digestibility when the diets were fed to young pigs from 13 to $23 \mathrm{~d}$ of age, confirming that DGE is a satisfactory indicator of fatty-acid digestibility.

2. Analyses of the fatty acids in the digesta removed from various levels of the digestive tract of the piglets showed a progressive increase in the ratio of stearic to octadecenoic acid.

3. Calculation of the quantities of fatty acids derived from the diet which were present in the different regions of the digestive tract showed that stearic acid was absorbed from the small intestine, although octadecenoic acid was absorbed preferentially.

4. Comparisons of the amounts of stearic and octadeccnoic acids in the ileal and colonic digesta derived from $x \mathrm{~kg}$ diet showed that the disappearance of octadecenoic and octadecadienoic acids was matched by the appearance of stearic acid, which suggests that biohydrogenation was occurring.

5. Consideration of the effect of the microflora on the unabsorbed residues of dietary fat suggests that the factors affecting fat digestion cannot adequately be examined by mcasuring either apparent or corrected fatty acid digestibilities.
\end{abstract}

The inverse relationship between melting points of fats and their digestibilities has long been recognized (Deuel \& Holmes, r921). Carroll (1958) showed that the longchain saturated fatty acids of high melting points were poorly digested when given as free fatty acids to rats, in contrast to saturated long-chain acids and short-chain saturated fatty acids which have lower melting points. Renner \& Hill (I96I) showed that, in the chick, the long-chain saturated fatty acids in natural fats had lower digestibilities than the unsaturated fatty acids. Similar effects were observed in the pig by Bayley \& Lewis (1965) and by Hamilton \& McDonald (1969), and in the pre-ruminant lamb by Walker \& Stokes (1970). However, the interactions which occur during digestion between individual fatty acids (Freeman, I969) and the effects of the intramolecular disposition of the fatty acids in the triglyceride molecules on fat digestion (Mattson, 1959; Davis \& Lewis, 1969) mean that the digestibility of a fat is not a simple function of its fatty-acid composition.

Studies of fat digestion based upon comparisons of dietary intakes and faecal outputs confound at least three processes: the emulsification and absorption of fatty acids in the small intestine, the addition of fat of endogenous origin to the digesta, and modifications of unabsorbed fat residues in the large intestine. Gompertz \& Sammons (1963) attributed the higher proportion of stearic acid in the faecal fat than in the dietary fat to preferential absorption of the unsaturated fatty acids in man, whereas Watson (1965)

\footnotetext{
* Present address: Chemcell Ltd, Edmonton, Alberta, Canada.
} 
concluded that biohydrogenation of the unsaturated fatty acids in the large intestine of man must be a significant factor in causing the high proportion of stearic acid in the faeces, since the ratio of stearic to oleic acid in the faeces could be modified by including an antibiotic in the diet. Carlson \& Bayley (1968) showed that saturation occurred in the large intestine of normal piglets, but not in gnotobiotic piglets, supporting the conclusion that at least a part of the stearic acid in the faeces is formed by hydrogenation of the unsaturated eighteen-carbon atom fatty acids.

The study reported here was designed to compare the amounts and types of fatty acids in digesta taken from different regions of the digestive tract with the amounts consumed in the diet, on a quantitative basis using tridodecyl glyceryl ether (DGE) as an absorption indicator (Carlson \& Bayley, 1972). These comparisons would provide information on the fate of the fatty acids which escape absorption from the small intestine and would thus provide an indication of the relative importance of selective absorption and of hydrogenation as reasons for the high proportion of stearic acid in the faecal lipids. This information is required to allow the usefulness of determining the digestibilities of individual fatty acids, by comparisons of diet and faeces, to be assessed.

\section{EXPERIMENTAL}

\section{Animals and diets}

Twenty-four piglets were weaned at $7 \mathrm{~d}$ of age and placed in individual cages. There were three diets based upon that described by Carlson \& Bayley (1972): a low-fat diet and two diets containing $10 \%$ of beef tallow. In the low-fat diet, 10\% of glucose replaced the beef tallow. All three diets contained $0.25 \%$ chromium sesquioxide (chromic oxide, $\mathrm{Cr}_{2} \mathrm{O}_{3}$ ) and the low-fat diet, and one of the high-fat diets contained $0.10 \%$ DGE. Eight piglets received each diet $a d$ lib., and the food intake and growth of the pigs were measured. The faeces voided from the $13^{\text {th }}$ to $23^{\text {rd }}$ day of age were collected, frozen and freeze-dried. At $23 \mathrm{~d}$ of age the pigs were killed and the contents of the stomach, the mid fifth of the length of the small intestine, the distal fifth of the small intestine (taken as representing jejunal and ileal digesta respectively), and the proximal part of the colon were removed, frozen under liquid nitrogen and freeze-dried.

\section{Determination of total fatty acids, fatty-acid composition and DGE}

The total fatty acids were extracted from samples of diet, digesta and faeces. The DGE in the samples was determined on aliquots of these extracts, and the composition of the fatty acids in the extracts were examined on pooled aliquots from the eight pigs in each diet. The geometrical and positional isomers of the octadecenoic and octadecadienoic acids were not separated although there are several of these in each fraction in beef tallow and in the lower part of the digestive tract. The dry-matter and $\mathrm{Cr}_{2} \mathrm{O}_{3}$ contents of the samples were also determined. All the analytical procedures are described by Carlson \& Bayley (1972). 
Table I. Food consumption, weight gain and apparent digestibility of dry matter for pigs between $\mathrm{I} 3$ and $23 d$ of age

(Mean values with their standard errors for eight pigs/diet)

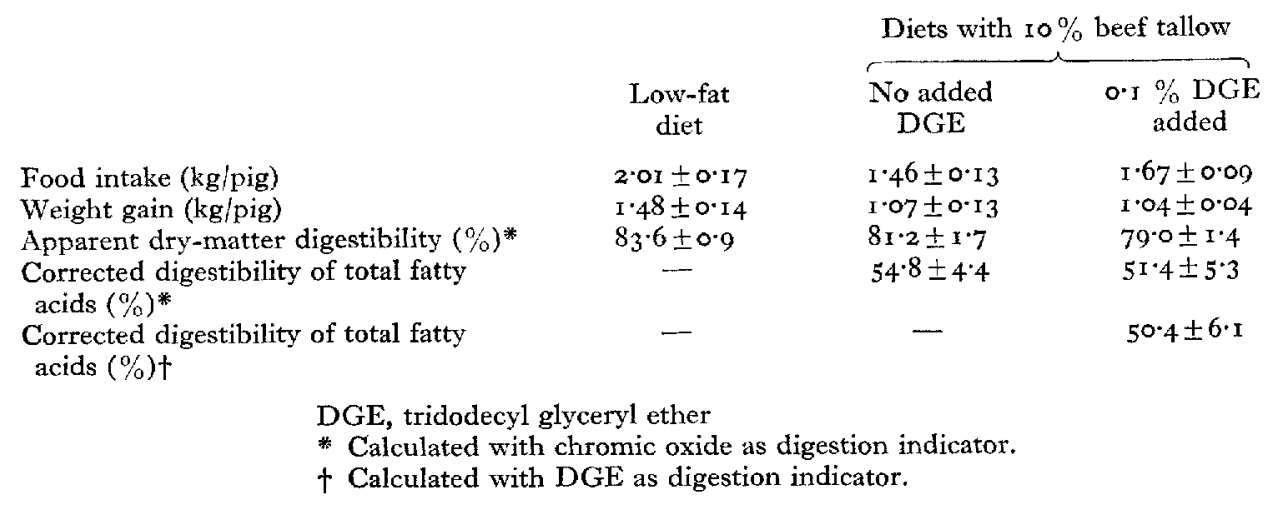

RESULTS

Substitution of Io\% beef tallow for glucose resulted in significant $(P<0.05)$ reductions in food intake and weight gains between $\mathrm{I} 3$ and $23 \mathrm{~d}$ of age ('Table $\mathrm{I}$ ), and the apparent digestibilities of the diets containing the beef tallow were lower than that of the low-fat diet. The addition of DGE did not influence food intake, weight gain or total digestibility of the beef-tallow diet, confirming the previous observation (Carlson \& Bayley, I972) that when added to the diet at $0.1 \%$ the DGE was nutritionally inert. The digestibilities of the total fatty acids in the beef-tallow diets were calculated with $\mathrm{Cr}_{2} \mathrm{O}_{3}$ as a digestion indicator, and the values were 'corrected', making the two assumptions commonly used in such determinations: it was assumed that the fatty acids voided in the faeces by the piglets receiving the low-fat diet represented the unabsorbed residues of the endogenous fatty acids added to the digestive tract, and that the small amount of fatty acid in the diet itself either was completely absorbed, or made an insignificant contribution to the faecal fat. The addition of the DGE to one of the diets did not affect the digestibility of the total fatty acids. The 'corrected' digestibility of the total fatty acids calculated with the DGE as digestion indicator was very similar to that calculated with $\mathrm{Cr}_{2} \mathrm{O}_{3}$ as the indicator, confirming the observations in the previous paper (Carlson \& Bayley, I972).

Comparison of the ratios of total fatty acids to either $\mathrm{Cr}_{2} \mathrm{O}_{3}$ or DGE in the diet and in the digesta samples or faeces allowed the amounts of fatty acid present in the digesta to be compared with the amount consumed in the diet. The values in Table 2 show the progressive absorption of the dietary fatty acids in passing through the digestive tract. The calculation based upon the ratio of total fatty acids to $\mathrm{Cr}_{2} \mathrm{O}_{3}$ suggests that there was a net secretion of fatty acids into the stomach, but earlier observations (Carlson \& Bayley, I968, I972) suggested that this was due to the $\mathrm{Cr}_{2} \mathrm{O}_{3}$ separating from the fatty acids in the stomach and moving into the small intestine ahead of the fatty acids. Thus, meaningful absorbabilities of the fatty acids from the regions of the digestive tract 
Table 2. Total fatty acids $(\mathrm{g})$ in $\mathrm{I} \mathrm{kg}$ of diet and in the digesta* derived from it for pigs of $23 d$ of age (faeces mean for 13 to $23 d$ of age)

(Mean values for eight pigs/diet)

\begin{tabular}{|c|c|c|c|c|}
\hline & \multicolumn{2}{|c|}{$\mathrm{Cr}_{2} \mathrm{O}_{3}$ as indicator } & \multicolumn{2}{|c|}{ DGE as indicator } \\
\hline & $\begin{array}{c}\text { Low-fat } \\
\text { diet }\end{array}$ & $\begin{array}{c}\text { Beef-tallow } \\
\text { diet }\end{array}$ & $\begin{array}{c}\text { Low-fat } \\
\text { diet }\end{array}$ & $\begin{array}{c}\text { Beef-tallow } \\
\text { diet }\end{array}$ \\
\hline Diet & $2 \cdot 0$ & $105 \cdot 5$ & $2 \cdot 0$ & $105 \cdot 5$ \\
\hline Stomach & $4.4 \pm 0.6$ & $139.4 \pm 5.1$ & $3 \cdot 6 \pm 0 \cdot 5$ & $106 \cdot 7 \pm 8 \cdot 2$ \\
\hline Jejunum & $2 \cdot 7 \pm 0 \cdot 4$ & $105.9 \pm 0.2$ & $2.4 \pm 0.5$ & $75 \cdot 2 \pm 7.8$ \\
\hline Ileum & $I \cdot 0 \pm 0.1$ & $54 \cdot 9 \pm 8 \cdot 5$ & $0.7 \pm 0.1$ & $40.0 \pm 4.8$ \\
\hline Colon & $4 \cdot 3 \pm 0 \cdot 6$ & $5 x \cdot 6 \pm 5 \cdot 2$ & $2 \cdot 2 \pm 0.2$ & $38 \cdot 9 \pm 3 \cdot 9$ \\
\hline Faeces & $5 \cdot 7 \pm 0.3$ & $57 \cdot 0 \pm 5 \cdot 6$ & $3.5 \pm 0.2$ & $56 \cdot 5 \pm 6 \cdot 7$ \\
\hline
\end{tabular}

distal to the stomach could not be calculated with $\mathrm{Cr}_{2} \mathrm{O}_{3}$ as an absorption indicator. In contrast, the calculations based upon the use of DGE show no change in the amount of fatty acid equivalent to $\mathrm{I} \mathrm{kg}$ diet as the digesta passed through the stomach, but showed a progressive net removal of fatty acids from the digesta, for samples taken from successively lower regions of the small intestine. The standard error of the mean amount of total fatty acid in the jejunum derived from $\mathrm{I} \mathrm{kg}$ of the beef tallow diet calculated using $\mathrm{Cr}_{2} \mathrm{O}_{3}$ as indicator was much greater than that calculated using DGE as indicator. This observation supports the conclusion that the $\mathrm{Cr}_{2} \mathrm{O}_{3}$ had separated from the fatty acids.

There appeared to be slightly less fatty acid in the digesta equivalent to I $\mathrm{kg}$ diet taken from the colon than from the cnd of the ileum, but this effect was not significant. However, there was much more fatty acid in the faeces than in the equivalent amount of ileal digesta, but the faeces were collected between ${ }^{3} 3$ and $23 \mathrm{~d}$ of age, whereas the digesta samples were removed at $23 \mathrm{~d}$ of age. Thus the greater quantity of fatty acids in the faeces than in the ileal digesta may have been due to the piglets being less able to digest the beef tallow in the earlier part of the experiment, or it could have been due to a net addition of fatty acids to the digestive tract distal to the proximal colon.

There were corresponding changes in the amount of fatty acids derived from $\mathrm{I} \mathrm{kg}$ of the low-fat diet for the digesta taken from successively lower regions of the small intestine. The progressive decrease in the amount of total fatty acid when the content of the stomach are compared to the contents of the jejunum and of the ileum, and the smaller quantity of total fatty acids in the ileum compared to the diet, suggest that any fatty acids of endogenous origin secreted into the small intestine must be reabsorbed to a much greater extent than examination of the faeces derived from a low-fat diet would suggest, and confirms the assumption that the small amount of fatty acid in the low-fat diet is well absorbed. However, the apparent net addition of fatty acids to the digesta as it passes through the lower part of the digestive tract thus raises some doubt 
Table 3. Composition of fatty acids (major components as \% of total fatty acids) in the diet and faeces of pigs between $\mathrm{I}_{3}$ and 23 d of age and in digesta taken at 23 d of age

(Pooled values from eight pigs/diet)

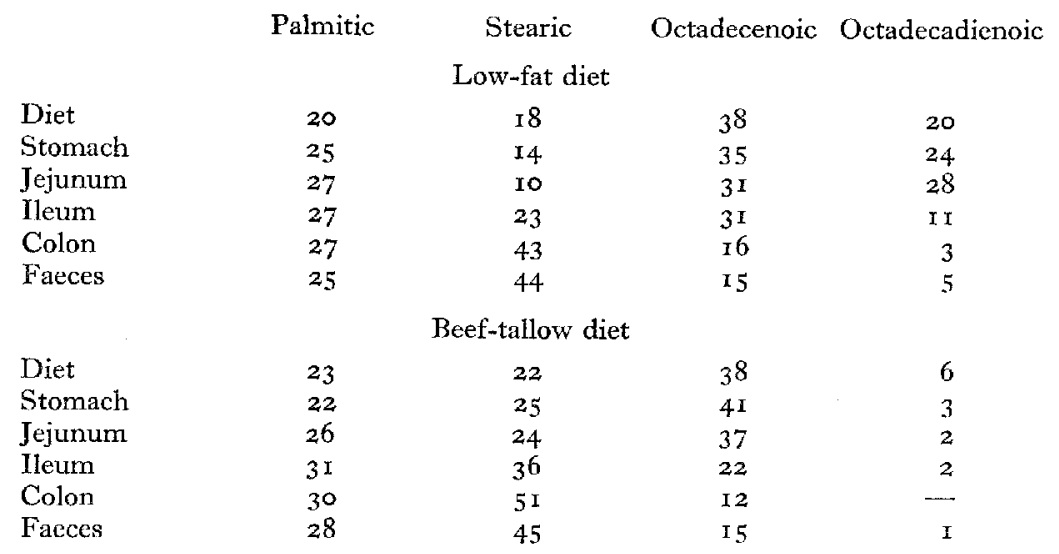

as to the validity of using faecal fat outputs from low-fat diets to correct fatty acid digestibilities on high-fat diets.

The proportions of the major component fatty acids in the total fatty acids extracted from the diet, digesta and faeces are shown in Table 3. The proportions of the saturated acids; palmitic and stearic, were greater in the digesta taken from successively lower regions of the digestive tract; stearic acid increased from $22 \%$ of the total in the beef tallow diet to $5 \mathrm{I} \%$ of the total in the colon contents derived from this diet. The composition of the fatty acids in the faeces were similar to those in the contents of the colon. Such changes in the pattern of the fatty acids in digesta as it passes through the small intestine could be due to the selective uptake of unsaturated fatty acids; unsaturated fatty acids are preferentially incorporated into the micellar phase (Freeman, 1969) and the capacity of the piglct to absorb micellar lipid exceeds the normal rate of micelle formation (Freeman, Noakes, Annison \& Hill, r968). However, the continued change in fatty-acid composition of the fat in the large intestine could be due to addition of saturated fatty acids to the digesta in the lower region of the digestive tract, as well as to the hydrogenation of the unsaturated fatty acids shown to be associated with the microflora of the large intestine (Carlson \& Bayley, I968).

\section{DISCUSSION}

The use of an indicator of fat absorption allows the extension of qualitative studies of fatty-acid patterns of digesta lipids to quantitative estimations of the flows of individual fatty acids through the digestive tract (Fig. r).

The results in Tables 2 and 3 have been used to calculate the amounts of eighteencarbon atom fatty acids in I $\mathrm{kg}$ of the beef-tallow diet, and in the digesta and faeces derived from it. The upper part of the figure shows that the amounts of these unsaturated fatty acids in the digesta were less in the jejunum and ileum, than in I kg of 

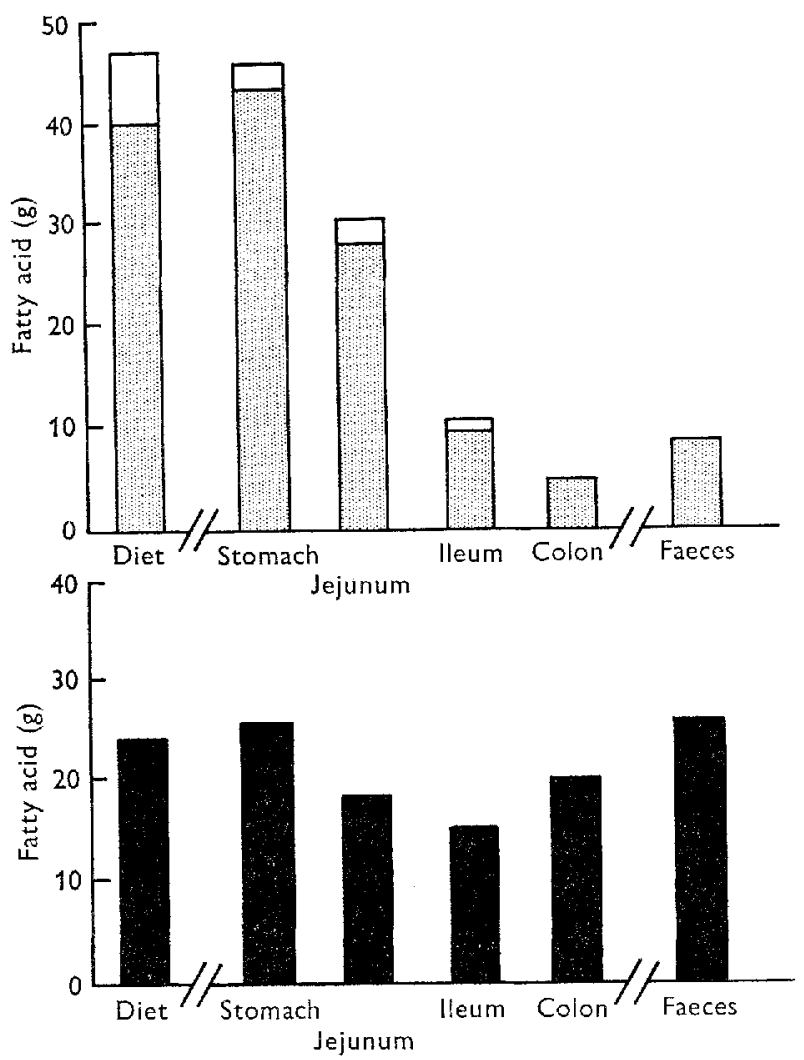

Fig. I. Amounts of octadecadienoic, octacedenoic and stearic acids in $\mathrm{r} \mathrm{kg}$ of the diet containing $10 \%$ beef tallow, and the amounts of these acids, derived from $\mathrm{I} \mathrm{kg}$ of diet, in the digesta and faeces of pigs between $I 3$ and $23 \mathrm{~d}$ of age. $\square$, octadecadienoic acid; 国, octadecenoic acid; stearic acid.

diet, or in the stomach contents derived from it. This is consistent with preferential uptake of these fatty acids from the small intestine. The continued reduction in the amount of these unsaturated fatty acids, when equivalent amounts of digesta from the colon and ileum are compared, indicates a net disappearance of unsaturated fatty acids. The lower part of Fig. I shows the amounts of stearic acid presented on the same basis as for the unsaturated acids. There was less stearic acid in the digesta taken from the jejunum or ileum than in an equivalent amount of the diet or stomach contents, indicating an actual absorption of stearic acid from the beef tallow by the piglets. However, there is a greater quantity of stearic acid in the digesta from the colon than in an equivalent amount of ileal digesta, indicating either stearic acid secretion into the large intestine or saturation of the unabsorbed octadecenoic acid which flowed from the small intestine into the large intestine. It is noteworthy that the increase in the amount of stearic acid $(0.5 \mathrm{~g})$ which occurred between equivalent amounts of ileal and colonic digesta was almost equal to the decrease in the amounts of unsaturated eighteen-carbon atom fatty acids $(0.6 \mathrm{~g})$ which occurred between equivalent amounts of ileal and colonic digesta, providing quantitative evidence of the importance of the 
Table 4. Apparent and corrected fatty-acid absorbabilities and digestibilities calculated from analyses of ileal digesta and faeces of pigs between 13 and $23 d$ of age

\begin{tabular}{|c|c|c|c|c|}
\hline & \multicolumn{2}{|c|}{ Absorbability (ileal contents) } & \multicolumn{2}{|c|}{ Digestibility (faeces) } \\
\hline & Apparent & Corrected* & Apparent & Corrected* \\
\hline Total fatty acids & $62 \cdot 1(4 \cdot 5) \dagger$ & $62 \cdot 7(4 \cdot 5) \dagger$ & $46 \cdot 4(6 \cdot 3) t$ & $50.4(6.6) \dagger$ \\
\hline Palmitic acid & $49 \cdot I$ & $49 * 9$ & $34 \cdot 8$ & $3^{8 \cdot 4}$ \\
\hline Stearic acid & $3^{8 \cdot 2}$ & $38 \cdot 9$ & -9.5 & $-2 \cdot 9$ \\
\hline Oleic acid & $78 \cdot 1$ & $78 \cdot 6$ & $78 \cdot 9$ & $80 \cdot 2$ \\
\hline Linoleic acid & $87 \cdot 4$ & $88 \cdot 6$ & $9 I \cdot I$ & $93 \cdot 8$ \\
\hline
\end{tabular}

* Corrected absorbabilities and digestibilities, calculated by assuming that the fatty acids in the ileal digesta and facces from the pigs receiving the low-fat diet were of endogenous origin, i.e. that the fatty acids in the low-fat diet were completely absorbed.

$\uparrow$ Standard error of the mean for eight pigs.

role of the microflora in affecting the results of fatty-acid balance studies. The faeces contained more octadecenoic and stearic acid than equivalent amounts of colonic digesta but, since these samples of faeces were not obtained in the same manner as the digesta samples, this observation merely supports the conclusions that either the piglets were not absorbing the fatty acids as completely throughout the balance period as they were on the final day or that there was a net addition of fatty acids to the contents of the large intestine.

The modifications of the fatty acids in the digestive tract, which occurred after the digesta had passed beyond the region where fatty-acid absorption is believed to be complete, cast doubt on the validity of using digestibilities of individual fatty acids as measures of the extent to which they can be absorbed from different fats by the animal. The ileal absorbabilities of the fatty acids from the beef tallow determined after killing the piglets of $23 \mathrm{~d}$ of age were much greater for the total and for the saturated fatty acids than the digestibilities determined conventionally, by collecting the faeces between the $x_{3}$ th and $23^{\text {rd }} \mathrm{d}$ of age. 'Correction' of the ileal absorbabilities made little difference to the values because of the small amounts of fatty acid in the ileal digesta of the piglets receiving the low-fat diet (Table 2).

This observation supports the assumption that the small amounts of fatty acid present in a low-fat diet used to allow 'correction' of fatty acid digestibility values is completely absorbed and thus the corrected ileal absorbability values were so similar to the apparent that there is little justification for the calculation. In contrast, the appreciable quantities of fatty acids in the faeces derived from the low-fat diet mean that 'corrected' digestibility values are higher than the apparent digestibility values. However, the biological significance of the correction of digestibility values using the quantities of fatty acid added to the digesta in the lower part of the digestive tract is doubtful.

The results of this experiment provide evidence for the hydrogenation of unsaturated, eighteen-carbon fatty acids in the lower part of the digestive tract, thus supporting the conclusion of Watson (1965) and of Carlson \& Bayley (I968) that an appreciable part of the stearic acid in the faeces is formed by hydrogenation. This conclusion contrasts with that of Gompertz \& Sammons (I963), who basec 
conclusion on the absence of hydrogenation of erucic acid as it passed through the large intestine of man. Freeman, Holme \& Annison (1968) produced direct evidence of biohydrogenation of oleic acid in the digestive tract of the piglet using ${ }^{14} \mathrm{C}$-labelled oleic acid; these authors also showed that the extent of this hydrogenation was dependent upon the type of fat in the diet. 'Therefore the determination of either 'apparent' or 'corrected' digestibilities of the individual fatty acids in natural foods must be considered to be of limited usefulness in critical experiments designed to study the factors limiting fatty acid utilization by comparing food intake and faecal output. 'The objects of such studies would be better served by direct examinations of small-intestinal digestion, such as those made by Freeman, Noakes et al. (1968) and such investigations could be made quantitative by the use of a fat-soluble marker, such as the DGE described in this report.

This work was supported by the Canada Department of Agriculture.

\section{REFERENCES}

Bayley, H. S. \& Lewis, D. (1965). F. agric. Sci, Camb. 64, 373.

Carlson, W. E. \& Bayley, H. S. (r968). Can. F. Anim. Sci. 48, 3 I5.

Carlson, W. E. \& Bayley, H. S. (1972). Br. F. Nutr. 28, 295 .

Carroll, K. K. (1958). F. Nutr. 64, 399.

Davis, R. H. \& Lewis, D. (1 969). O. agric. Sci., Camb. 72, 217.

Deuel, H. J. Jr \& Holmes, A. D. (192,1). Am. F. Physiol. 54, 479.

Frceman, C. P. (1969). Br. F. Nutr. 23, 249.

Freeman, C. P., Holme, D. W. \& Annison, E. F. (1968). Br. F. Nutr. 22, 65 r.

Freeman, C. P., Noakes, D. E., Annison, E. F. \& IHill, K. J. (1968). Br. F. Nutr, 22, 739 .

Gompertz, S. M. \& Sammons, H. G. (I963). Clinica chim. Acta 8, 59 I.

Hamilton, R. M. G. \& McDonald, B. E. (1969). J. Nutr. 97, 33.

Mattson, F. H. (1959). \%. Nutr. 69, 338.

Renner, R. \& Hill, F. W. (1961). F. Nutr. 74, 254.

Walker, D. M. \& Stokes, G. B. (I970). Br. F. Nutr. 24, 425.

Watson, W. C. (1965). Clinica chim. Acta 12, 340. 